immunocompetence by assessing function of selected cell types involved in host resistance.

Methods Basal NK cell activity, lymphoproliferation following stimulation with anti-CD3, pokeweedmitogen or concanavalin A, and antibody forming cell response (AFC) were determined in splenocytes from female rats $(\mathrm{F}-344 ; \mathrm{N}=12$ per group) treated with placebo, IL-1ra alone (20 or $200 \mathrm{mg} / \mathrm{kg}$, SC daily), sTNF$\mathrm{RI}$ alone $(5$ or $50 \mathrm{mg} / \mathrm{kg}$, SC twice weekly) or the dose combinations of the two cytokine inhibitors for 21 days. Satellite groups received positive control agents for the NK cell assay $(0.5 \mathrm{~mL}$ anti-asialo GM 1 antibody) or the mitogenesis and AFC assay (25 mg/kg cyclophosphamide).

Results Basal NK cell response was increased 21\% ( $\mathrm{p}<0.05$ ) over placebo control only at the combination of $200 \mathrm{mg} / \mathrm{kg}$ IL$1 \mathrm{ra}+5 \mathrm{mg} / \mathrm{kg}$ sTNF-RI. No effect was noted with IL-1ra or TNF-RI alone or at higher combination doses. Cells from IL-1ra $(200 \mathrm{mg} / \mathrm{kg})$ treated animals showed minimal decreases $(30 \%, \mathrm{p}$ $<0.05)$ on lymphocyte proliferation only in response to anti CD3-mediated proliferation. Statistically significant differences $(+10$ to $-48 \%)$ in proliferative activity to all mitogens were observed with sTNF-RI alone or in combination with IL1-ra, but were not dose related. Diminution in the AFC response (29$36 \%)$ was noted for sTNF-RI alone $(50 \mathrm{mg} / \mathrm{kg})$ or in combination with both Il-1ra doses compared to the control group; however the differences were not statistically significant. IL1-ra alone, the low dose of sTNF-RI $(5 \mathrm{mg} / \mathrm{kg}$ ) alone or in combination with IL-1ra doses had no significant effect on AFC response.

Conclusion Thus, IL-1ra alone has minimal effects on selected functions of the immune system. Effects on immune function noted at $50 \mathrm{mg} / \mathrm{kg}$ sTNF-RI were considered minimal and importantly were not exacerbated by the combination of IL-1ra.

\section{THU0097 THE DISCREPANCY BETWEEN MRNA AND PROTEIN EXPRESSION OF THE TUMOUR-SUPPRESSOR GENE MASPIN IN SYNOVIAL TISSUE MIGHT CONTRIBUTE TO SYNOVIAL HYPERPLASIA IN RHEUMATOID ARTHRITIS (RA)}

1J Schedel, 'E Jeisy Walder, ${ }^{2}$ B Simmen, ${ }^{1}$ BA Michel, 'RE Gay, 'S Gay. 'Center of Experimental Rheumatology, Department of Rheumatology, University Hospital of Zurich; ${ }^{2}$ Department of Orthopedic Surgery, Schulthess Clinics, Zurich, Switzerland

10.1136/annrheumdis-2001.974

Background RA is a chronic disease characterised by tumour-like features such as synovial hyperplasia, invasive growth of the synovium and joint destruction. Maspin is a member of the serpin family of protease inhibitors with gene sequence similarities to plasminogen activator inhibitor. Maspin exhibits also tumoursuppressor activity by inhibiting cell motility, invasion and metastasis, and is down-regulated in breast and prostate cancer.

Objectives We investigated the expression of maspin in RA synovial tissue and compared it with the expression in osteoarthritis (OA) and normal synovial tissues (NS).

Methods The expression of maspin was determined in synovial tissue samples obtained during synovectomy or arthroplastic surgery. Using specific primers for maspin, a $237 \mathrm{bp}$ fragment was amplified from cDNA obtained from cultured RA (6), OA (4) and normal (1) synovial fibroblasts (SF) by reverse transcription polymerase chain reaction (RT-PCR). In addition, mRNA expression levels were determined quantitatively by real-time PCR (TaqMan). Using non-radioactive in situ hybridization, mRNA expression of maspin was investigated using snap-frozen and paraffin sections. To identify the cell types expressing maspin, immunohistochemistry was performed on serial tissue sections using monoclonal antibodies against CD3 (T cells) and CD68 (macrophages). SDS-PAGE and Western blotting was performed using anti-maspin antibodies to evaluate the protein expression in total synovial tissue and in cultured synovial fibroblasts (3rd 4th passage). Protein from normal breast tissue was used as positive control.

Results RT-PCR revealed expression of maspin in all cDNA samples from cultured synovial fibroblasts. By real-time PCR, maspin mRNA was found 2-fold decreased in RA-SF compared to OASF and 70-fold in comparison to NS-SF. Using in situ hybridization, maspin mRNA was expressed in RA as well as in OA and normal synovial tissue. Whereas in normal and OA samples, expression of maspin mRNA was restricted to synovial fibroblasts in the synovial lining and sublining, in RA, maspin could additionally be observed within perivascular infiltrates in mononuclear and multinucleated cells. Importantly, maspin transcripts were also found at sites of invasion into cartilage and bone. Interestingly, on the protein level, maspin could only be detected in normal breast tissue, but neither in RA synovial tissue nor in RA or OA synovial fibroblasts.

Conclusion Since maspin is not expressed in RA-SF on the protein level, it is hypothesised that the lack of maspin could contribute to the hyperplasia of the synovial tissue in RA.

\section{THU0098 MODIFICATION OF T-LYMPHOCYTES SUBSETS IN RHEUMATHOID ARTHRITIS}

B Mioara, A Isac. Department of Rheumatology and Rehabilitation, University of Medicine and Pharmacy Timisoara Romania, Timisoara, Romania

\subsection{6/annrheumdis-2001.975}

Background The role of T-lymphocytes (LT) in the pathogenesis of rheumathoid arthritis (RA) is demonstrated, but the site of Tcell activation is still unknown. The differentiation and maturation of LT took place either in the peripheral blood (PB) and synovial fluid (SF). This process can be followed using the isoforms of the leucocyte common antigen (CD45). Cells expressing CD45RA demonstrate functional characteristics of "naive cells" and those expressing CD45RO of "memory cells". Following stimulation, naive cells lose CD45 RA and gain CD45 RO with a transitional stage of dual CD45RA and CD45RO positivity. In RA, the LT from the PB express primarily CD 45RA whereas most LT found in the SF express CD45RO. Expression of HLA-DR is increased on LT found in SF. The population of dual positive LT cells CD45RA+CD45RO+ is expanded in SF, supporting the hypothesis that the LT are activated within the joint.

Objectives Using monoclonal antibodies labelled with fluorochromes and the flow-cytometry technique we tried to evidentiate the most important modification of lymphocytes subtypes in $\mathrm{PB}$ and SF in patients with chronic arthritis.

Methods The study was performed on 29 cases: 19 with RA in the stage II and III of evolution; 8 cases with ankylosing spondylitis and 2 cases with knee ostheoarthritis used as control. Paired samples of PB and SF were obtained from all the patients. From these, nucleated cells were prepaired (after erithrocyte lysis in $\mathrm{PB}$ ) and mononuclear cells in SF after density gradient centifugation. The cells from both sources were labelled with monoclonal antibodies conjugated with fluorochromes in three colour 